\title{
Fragility Fracture Care: An Urgent Need to Implement the Integrated Model of Geriatric Care
}

\author{
Jae-Young Lim \\ Department of Rehabilitation Medicine, Seoul National University Bundang Hospital, Seoul National University College of Medicine, \\ Seongnam, Korea
}

On January 24, 2019, a member of the National Assembly held a public hearing in the National Assembly Members' Office. This public hearing was aimed at promoting the development of a comprehensive integrated fragility fracture care system for geriatric patients. The hearing involved active discussions and suggestions for the prevention of fragility fractures, multidisciplinary care, and rehabilitation.

Osteoporotic fracture or fragility fracture has been recognized as a geriatric disease manifesting with various and complex problems that have a significant detrimental effect on whole-body condition and function as well as musculoskeletal problems due to the fracture itself. ${ }^{1)}$ The term "geriatric fracture" has been also used to address the nature of fragility fractures, that can be more easily understood, and is better to attract public awareness. ${ }^{2)}$ The annual incidence of fragility fractures exceeds 150 hip fractures per 100,000 people in the most developed countries. ${ }^{3)}$ Moreover, there have been rapid increases in hip fracture rates in several major countries with high populations, such as China and India. In Korea, the incidence of hip fractures continues to increase. ${ }^{4)}$ Regarding the functional trajectory throughout life, frail older adults are vulnerable to fall and fall-related injuries. They may experience a severe functional decline or may be unable to survive; however, with proper management, some older adults are able to return to their previous levels of function. Fall-related fractures in late life may threaten independence, sometimes to levels below the disability threshold. Partially recovered patients often have a lower functional state. Repeated fall and related fractures could lead to a more severe functional decline or even death. However, successful fragility fracture care allows patients to return to their previous levels of functioning. In an ideal scenario, although repeated falls and fractures may occur, older adults could maintain their original functional capacities.

Diverse components can be targeted for fragility fracture care, with the main goal of restoring the previous level of function and preventing repeated fractures. ${ }^{5)}$ Fragility fractures are a geriatric disease with multiple risk factors and comorbidities. Therefore, we need a multidisciplinary approach to achieve a good outcome after a fragility fracture. However, we face several barriers in fragility fracture care. ${ }^{6}$ For example, there are substantial gaps between service delivery and practice. Patient barriers also exist. Most patients and their families have low levels of awareness of post-acute care. Insufficient medical and surgical leadership and lacking hospital administration support are also problems. Furthermore, integrated care systems involving orthopedic surgeons, geriatricians, and rehabilitation specialists are quite limited. Therefore, communication and collaboration do not work well in real world clinical practice.

Fracture Liaison Services (FLS) are systems developed to ensure that fracture risk assessment and treatment are provided to all patients with fragility fractures. ${ }^{7)}$ FLS have been implemented in many countries as standard care for fragility fractures. International initiatives on fragility fracture care to promote FLS in each country or region have emerged. The Fragility Fracture Network (FFN) is a leading global initiative aimed at promoting multidisciplinary management and secondary prevention among patients with fragility fractures. The FFN declared a Global Call to Action on fragility fractures to improve the care of people with fragility fractures. ${ }^{8)}$ There is an urgent need to improve: 1) acute multidisciplinary care for individuals with hip, clinical vertebral, or other major fragility fractures; 2) rapid secondary prevention to prevent future fractures after the first occurrence of fragility fracture in both younger and older individuals; 3) ongoing post-acute care for individuals with impaired functional capacities caused by hip and major fragility fractures. More than 80 leading organizations worldwide in the fields of medicine and nursing for older people have endorsed the Global Call to Action. Recently, the Korean Geriatrics Society, the Korean Society for Bone and Mineral Research, and the Korean Academy of Rehabilitation Medicine have also endorsed the Global Call to Action. The FFN has translated the Global Call to Action into the world's major languages. 
The Korean language version of the Global Call to Action can now be downloaded via a webpage (http://www.fragilityfracturenetwork.org/cta). ${ }^{9)}$

A nationwide cohort of geriatric patients with fragility fractures supported by the National Health Clinical Research was established in 2016. This research project incorporating a cohort study has developed standardized fragility fracture integrated rehabilitation management (FIRM) based on the critical rehabilitation pathway for fragility fractures in both hospital and community settings. ${ }^{10,11)}$ As a Fracture Liaison Services based on rehabilitation, the FIRM was developed to fit the medical insurance and health care delivery system in Korea. In addition, the first FFN-Korea regional meeting endorsed by the Global FFN was held in October 2018 in Seoul. In late November 2018, the Asian Regional Expert Meeting was successfully held in Tokyo, Japan, which was a pivotal moment in enhancing the standards of fragility fracture care in many Asian countries.

The public hearing on fragility fracture care held in the National Assembly Members' Office in January 2019 included experts on fragility fracture care, policymakers, and citizens, and focused on the poor current care system for fragility fractures. This public hearing contributed to building a basic framework for an institutional or legislative support system, in order to support a multidisciplinary care model for the prevention of secondary fractures and comprehensive and integrated rehabilitation after a fragility fracture. Furthermore, knowledge and ideas based on geriatric medicine and research are imperative in establishing a customized care system for patients with fragility or geriatric fractures. Therefore, fragility fracture care will contribute to the promotion of the role of geriatric specialists as a core element in the integrated model of geriatric care.

\section{CONFLICTS OF INTEREST DISCLOSURES}

The author claims no conflicts of interest.

\section{REFERENCES}

1. Cifu DX, Lew HL, Oh-Park M. Geriatric rehabilitation. St. Louis: Elsevier Science Health Science; 2018.

2. Switzer JA, Bozic KJ, Kates SL. Geriatric fracture care: future trajectories: a 2015 AOA Critical Issues Symposium. J Bone Joint
Surg Am 2017;99:e40.

3. Kanis JA, Odén A, McCloskey EV, Johansson H, Wahl DA, Cooper C; IOF Working Group on Epidemiology and Quality of Life. A systematic review of hip fracture incidence and probability of fracture worldwide. Osteoporos Int 2012;23:2239-56.

4. Ha YC, Kim TY, Lee A, Lee YK, Kim HY, Kim JH, et al. Current trends and future projections of hip fracture in South Korea using nationwide claims data. Osteoporos Int 2016;27:2603-9.

5. Kang JH, Lee G, Kim KE, Lee YK, Lim JY. Determinants of functional outcomes using clinical pathways for rehabilitation after hip fracture surgery. Ann Geriatr Med Res 2018;22:26-32.

6. Kates SL, O'Malley N, Friedman SM, Mendelson DA. Barriers to implementation of an organized geriatric fracture program. Geriatr Orthop Surg Rehabil 2012;3:8-16.

7. McLellan AR, Wolowacz SE, Zimovetz EA, Beard SM, Lock S, McCrink L, et al. Fracture liaison services for the evaluation and management of patients with osteoporotic fracture: a cost-effectiveness evaluation based on data collected over 8 years of service provision. Osteoporos Int 2011;22:2083-98.

8. Dreinhöfer KE, Mitchell PJ, Bégué T, Cooper C, Costa ML, Falaschi $\mathrm{P}$, et al. A global call to action to improve the care of people with fragility fractures. Injury 2018;49:1393-7.

9. Fragility Fracture Network. Global call to action on fragility fractures achives unprecedented levels of support [Internet]. Zurich: Fragility Fracture Network; 2018 [cited 2019 Mar 17]. Avalible from: http://www.fragilityfracturenetwork.org/cta.

10. Lee SY, Beom J, Kim BR, Lim SK, Lim JY; Fragility Fracture Rehabilitation Study Group. Comparative effectiveness of fragility fracture integrated rehabilitation management for elderly individuals after hip fracture surgery: a study protocol for a multicenter randomized controlled trial. Medicine (Baltimore) 2018;97:e10763.

11. Lee SY, Beom J. Letter to the editor: specific and stepwise postoperative rehabilitation program is needed in the elderly after hip fracture surgery. Ann Geriatr Med Res 2016;20:233-4.

Corresponding author Jae-Young Lim, MD, PhD Department of Rehabilitation Medicine, Seoul National University Bundang Hospital, Seoul National University College of Medicine, 82 Gumi-ro 173beon-gil, Bundang-gu, Seongnam 13620, Korea E-mail: drlim1@snu.ac.kr ORCID code: https://orcid.org/0000-0002-9454-0344

Received: March 18, 2019 Accepted: March 19, 2019 